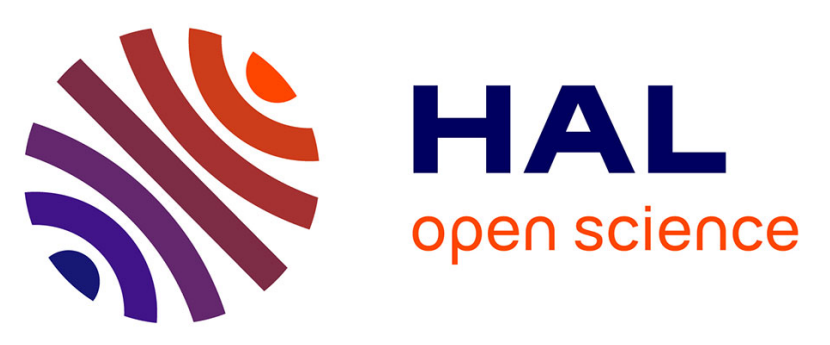

\title{
Optimization driven multi-hop network design and experimentation: the approach of the FP7 project OPNEX
}

Kostas Choumas, Stratos Keranidis, Thanasis Korakis, Iordanis Koutsopoulos, Leandros Tassiulas, Felix Juraschek, Mesut Günes, Emmanuel Baccelli, Pawel

Misiorek, Andrzej Szwabe, et al.

\section{To cite this version:}

Kostas Choumas, Stratos Keranidis, Thanasis Korakis, Iordanis Koutsopoulos, Leandros Tassiulas, et al.. Optimization driven multi-hop network design and experimentation: the approach of the FP7 project OPNEX. IEEE Communications Magazine, Jun 2012, United States. pp.122 - 130, 10.1109/MCOM.2012.6211496 . hal-00722397

\section{HAL Id: hal-00722397 https://hal.science/hal-00722397}

Submitted on 1 Aug 2012

HAL is a multi-disciplinary open access archive for the deposit and dissemination of scientific research documents, whether they are published or not. The documents may come from teaching and research institutions in France or abroad, or from public or private research centers.
L'archive ouverte pluridisciplinaire $\mathbf{H A L}$, est destinée au dépôt et à la diffusion de documents scientifiques de niveau recherche, publiés ou non, émanant des établissements d'enseignement et de recherche français ou étrangers, des laboratoires publics ou privés. 


\title{
Optimization driven Multi-Hop Network
}

\section{Design and Experimentation: The Approach of the FP7 Project OPNEX}

\author{
Kostas Choumas ${ }^{1}$, Stratos Keranidis ${ }^{1}$, Thanasis Korakis ${ }^{1}$, Iordanis Koutsopoulos ${ }^{1}$, Leandros \\ Tassiulas $^{1}$, Felix Juraschek ${ }^{2}$, Mesut Günes ${ }^{2}$, Emmanuel Baccelli ${ }^{3}$, Paweł Misiorek ${ }^{4}$, Andrzej \\ Szwabe $^{4}$, Theodoros Salonidis ${ }^{5}$, Henrik Lundgren ${ }^{5}$ \\ ${ }^{1}$ University of Thessaly and CERTH, Greece \\ ${ }^{2}$ Freie Universität Berlin (FUB), Germany \\ ${ }^{3}$ INRIA, France \\ ${ }^{4}$ Poznan University of Technology (PUT), Poland \\ ${ }^{5}$ Technicolor Lab, Paris, France
}

\begin{abstract}
The OPNEX project exemplifies system and optimization theory as the foundations for algorithms that provably maximize capacity of wireless networks. The algorithms termed in abstract network models have been converted to protocols and architectures practically applicable to wireless systems. A validation methodology through experimental protocol evaluation in real network testbeds has been proposed and used. OPNEX uses recent advances in system theoretic network control, including the Back-Pressure principle, max-weight scheduling, utility optimization, congestion control, and the primal-dual method for extracting network algorithms. These approaches exhibited vast potential for achieving high capacity and full exploitation of resources in abstract network models and found their way to reality in high performance architectures developed as a result of the research conducted within OPNEX.
\end{abstract}

\section{INTRODUCTION}

Over the last few decades, several theoretical underpinnings on systems control and optimization theory have been established that give rise to a novel spirit for network control and protocol architecting.

Contact author: Stratos Keranidis - Email: efkerani@uth.gr 
However, wireless networks predominantly operate based on principles and protocols inherited from their wire-line counterparts or rely on purely empirical, ad-hoc resource allocation and parameter adaptation rules. This comes also from the fact that optimization theory concepts fail to be translated to practical systems, due to the impractical assumptions they are based on.

The core objective of OPNEX project is to build the gap between theory and practice, through the adoption of a disruptive systems optimization and control approach, including the Back-Pressure $(B P)$ principle [1], [2] , max-weight scheduling, utility optimization, congestion control, and the primaldual method for designing architectures and protocols for wireless networks. The underlying principle of $B P$ policy, as depicted in Figure 1, is to prioritize in forwarding the use of links $(i, j)$ with higher products of link rates $\left(R_{i, j}\right)$ and backlog differentials, $\left(q_{i}-q_{j}\right)$, with $q_{i}$ the queue size of node $i$. The objective of the $B P$ policy is to determine the set of active links in order to maximize the total weighted short-term throughput, given by:

$$
T=\sum_{(i, j)}\left(q_{i}-q_{j}\right) R_{i, j}
$$

such that $R_{i j} \in \Lambda$, where $\Lambda$ is the region of feasible rate vectors dictated by system constraints.

In wireless networks, the fundamental constituent performance metrics are throughput, end-to-end delay and energy efficiency and there is already a big body of research pursuing them. In an effort to investigate performance improvement of different optimization driven approaches in terms of the aforementioned metrics, OPNEX project contributes by:

- proposing two different architectures that are based on the well-known max-weight $B P$ technique and target network throughput;

- considering the inherent drawback of $B P$-oriented approaches, which is the poor delay performance they experience, and proposes a delay-aware Network Utility Maximization (NUM) system;

- investigating the energy consumption of wireless networks and more specifically the case of wireless sensor networks (WSNs) in the context of environmental monitoring.

Inspired by the philosophy of $B P$, we propose a distributed load-aware routing protocol, where the next hop is selected based on a newly defined metric that is formed by both the queue lengths of nodes in the path from the source to the destination, as well as the corresponding link qualities. Moreover, we further contribute by introducing the XPRESS architecture, which is the first implemented scheme that integrates Network Utility Maximization (NUM) congestion control with $B P$ routing and centralized max-weight TDMA MAC scheduling and thus complements the aforementioned distributed protocol. Both of the $B P$ - 
inspired schemes are implemented and experimentally evaluated. The former one is evaluated in NITOS [3], which supports experimentation with 802.11 compatible devices, while the latter one is evaluated in Technicolor's testbed that is composed of custom TDMA MAC enabled devices.

Considering the poor delay performance of $B P$-based schemes, we propose the DANUM system, which is able to apply NUM-derived priorities to multi-class traffic with respect to both delay and rate per flow. The delay-awareness of the DANUM framework results from the definition of a new optimization variable, which models the delay-aware utility definitions for both TCP and UDP flows. The DANUM system is implemented and evaluated in wnPUT [4] and NITOS testbeds, which are both compatible with the 802.11 standard.

In the field of energy consumption we study the performance of WSNs, where massive amount of information needs to be circulated through the sensor nodes. We investigate the trade-off between energy usage and overall rate of successfully received messages, in experiments where a gossiping routing algorithm is used to transfer messages from the source nodes to the sink. An implementation that demonstrates energy efficiency through an environmental monitoring experiment was realized in DEStestbed [5], which is a full-fledged wireless sensor testbed that also provides for gathering of accurate energy consumption measurements.

The aforementioned protocols have resulted through optimization driven research and have all been implemented and experimentally validated under realistic settings. In order to provide for proper evaluation of the proposed schemes, four different realistic wireless testbeds were developed through the OPNEX project. More specifically, there were built two 802.11 compatible testbeds, a large-scale one in CERTH named NITOS and a small-scale one in PUT named wnPUT, one custom TDMA MAC based testbed was developed by Technicolor and one wireless sensor testbed was developed by FUB and named DES-testbed. In this paper, we describe the resulting protocols, details about each one of the developed testbeds and moreover present results obtained through experimentation of the proposed protocols in the corresponding testbed facilities.

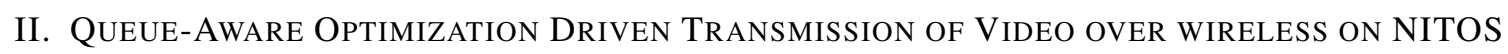
TESTBED

\section{A. QLR (Queue-Length aware Routing) protocol}

The efficiency of a multi-hop, mesh network is directly related to the routing protocol that is used for packet forwarding. A policy that achieves maximum throughput is the well-known $B P$ algorithm. One 
important outcome of the project was the Queue-Length aware Routing $(Q L R)$ routing protocol, which is inspired by the philosophy of $B P$.

The $Q L R$ protocol is based on the original implementation of the SRCR routing protocol used in Roofnet [6], an experimental Wireless Mesh deployment in MIT. While SRCR assigns ETT (Expected Transmission Time) weights to links, taking into account only link qualities, $Q L R$ considers queue levels of intermediate nodes as well, through the definition of the EPD (Expected Packet Delay) metric. More specifically, in $Q L R$, forwarder nodes identify the flow that each received packet belongs to and thereafter select the neighboring nodes that feature the minimum EPD value as the next hop. EPD metric evaluation is approximated as the product of the maximum internal queue length and the expected transmission time weight of the link that follows.

The implementation of our mechanism requires a proper signaling mechanism, so that periodical messages with $E P D$ info are propagated through the network, in a distributed way. Moreover, our scheme requires flow discrimination, which is not supported by the original SRCR protocol. To overcome this issue, we designed a dynamic structure of ordered queues, where each structure is used to store only packets associated with a specific known flow.

\section{B. NITOS-Testbed}

The experimentation environment that CERTH has developed for the purposes of OPNEX is NITOS testbed [3]. NITOS is a large-scale wireless testbed that currently consists of 40 operational Wi-Fi nodes. NITOS is a non-RF-isolated wireless testbed, outdoor deployed at the University of Thessaly campus. Users can perform their experiments by reserving slices (nodes, frequency spectrum) of the testbed through NITOS scheduler that together with the OMF management framework support ease of use for experimentation and code development.

\section{Experimentation Results}

A ring network consisting of five NITOS nodes has been designed, featuring a 2-hop and a 3-hop path as well. The experimental setup consists of an Iperf [7] client running at the source node, generating UDP traffic streams and an Iperf server residing at the destination node, receiving the generated data and collecting the overall statistics. We set the physical transmission rate for each node fixed to $6 \mathrm{Mbps}$ and use the frequency of $5280 \mathrm{MHz}$ to run our experiments in 802.11a mode, in order to avoid potential external interference. 
Figure 2 illustrates how the throughput achieved changes with respect to the traffic load injected in the network. We notice that the maximum throughput achieved for both schemes is $1.2 \mathrm{Mbps}$. Once the traffic load increases above the value of $1.2 \mathrm{Mbps}$, the system becomes unstable and both approaches invariably start to witness significant packet drop and throughput reduction. However, the $Q L R$ scheme manages to balance the load between the two available paths more efficiently, offering throughput performance nearly equal to the maximum value. On the other hand, the $S R C R$ scheme results in a continuous decrease of achieved throughput, as the traffic load increases up to the maximum value of $2 \mathrm{Mbps}$.

\section{Video Transmission Experimentation}

In this experiment, we use the same ring network to demonstrate the operation of video streaming applications over multi-hop wireless networks and particularly depict the benefits that the $Q L R$ protocol may offer in such scenarios. More specifically, we use a video of H.264 format that is transmitted from the source to the destination node. We manually adjust the appropriate video-bitrate, so that it allows for undeteriorated transmissions. Based on the results obtained from our previous experiment, we conclude that a typical value of traffic rate that can yield different performance in terms of throughput for the two approaches, is that of $2 \mathrm{Mbps}$. Due to this, we decided to encode the video under transmission with the exact video-bitrate value of 2 Mbps.

We use an external PC, which runs the client version of the $V L C$ platform to generate the traffic UDP stream at the application layer. Moreover, we run the server VLC version at the same PC to receive the corresponding traffic stream. The server machine of the NITOS testbed is used as the connecting part of the actual network and the external PC. All frames delivered from the PC to the source node, are forwarded to the destination node through the wireless part of the network. Finally, the frames delivered at the destination node are further delivered back to the external PC.

Eventually, we are able to compare the quality of the initially transmitted video and the video resulting from transmissions that follow the protocols under consideration. In Figure 3, two screen shots are provided that clearly depict the superiority that the $Q L R$ protocol achieves in terms of video quality. At the left hand side, we notice that the video-bitrate of the transmitted video cannot be supported by the network, which results in a distorted version of the original video. In contrary, we notice at the right hand side that the $Q L R$ protocol manages to balance the traffic between the two paths and as a result the video is delivered nearly unscathed. We have to note also that the average PSNR (Peak Signal-to-Noise Ratio) values of the two received videos are $32 \mathrm{~dB}$ and $13 \mathrm{~dB}$, for the $Q L R$ and SRCR schemes accordingly, where higher PSNR values correspond to video of higher quality. 


\section{CROSS-LAYER BACKPRESSURE ARCHITECTURE FOR WIRELESS MULTI-HOP NETWORKS ON}

\section{TECHNICOLOR's TESTBED}

\section{A. X-PRESS: Cross-layer Backpressure architecture for wireless multi-hop networks}

In this section, we summarize our contributions on the design of XPRESS, a throughput-optimal $B P$ architecture for wireless multi-hop networks, which is described in detail in [8]. XPRESS transforms a multi-hop wireless network to a wireless switch, where routing and scheduling decisions are made at packet time scale by a centralized backpressure scheduler. XPRESS is the first system that integrates NUM congestion control with backpressure routing and centralized max-weight TDMA MAC scheduling as it was originally proposed in [1].

XPRESS is composed of a mesh controller $(M C)$, which computes the optimal $B P$ schedule based on measured wireless network state, and the wireless network nodes, which measure the network state, perform congestion control and execute the computed schedule using a cross-layer protocol stack. The XPRESS cross-layer stack integrates the transport, network, and MAC layers. To achieve synergy among these layers on Technicolor's customized programmable 802.11 platform [9] required (i) a NUM congestion control mechanism to ensure the scheduler operates within the capacity region; (ii) a coordination mechanism between network-layer flow queues and MAC-layer link queues, which enables per-link queue implementation on memory-constrained wireless interfaces; and (iii) a multi-hop TDMA MAC protocol that ensures global synchronization among nodes and enables coordinated transmissions within slot boundaries according to the exact $B P$ schedule.

XPRESS nodes use an interference estimation mechanism of low measurement complexity (linear in number of network nodes) that allows the BP scheduler to determine at TDMA frame time scale which links can transmit without interference for all supported PHY data rates. The mechanism uses Received Signal Strength (RSS), complemented with an adaptive packet loss detection technique to cope with the RSS measurement limitations of 802.11.

At the mesh controller side, XPRESS reduces scheduling overhead using a novel speculative scheduling technique. This technique computes a schedule for a group of slots on a TDMA frame basis and performs the optimal BP computation for all slots in the frame based on speculated network queue state. In addition, we show that in our system the $B P$ computation at each slot reduces to a Maximum Weight Independent Set $(M W I S)$ computation in a binary conflict graph. This fact lowers computation complexity by bypassing exhaustive enumeration of all transmission possibilities. In the following, we give a brief summary of the XPRESS performance in our wireless testbed. 


\section{B. Technicolor Testbed}

The aforementioned protocol was designed, implemented and evaluated in the Technicolor wireless testbed, which is deployed in two locations of the Technicolor headquarters in Paris France. The deployment is a typical indoor office environment that spans three buildings and one partly covered parking garage, where nodes are deployed across four different floors. Each node is equipped with both off-theshelf wireless hardware, as well as Technicolor's customized programmable Wi-Fi cards and multi-sector antennas. A custom-made testbed management system is used for remote configuration and operation of the wireless nodes that run the Linux operating system.

\section{Testbed Evaluation}

In this section, we present experimental results that compare XPRESS to 802.11 DCF. For XPRESS, we fix the PHY rate for the data subframe to $24 \mathrm{Mbps}$. For $802.11 \mathrm{DCF}$ we use both a fixed $24 \mathrm{Mbps}$ PHY rate and the automatic PHY rate adaptation scheme of our Wi-Fi card (noted as auto-rate hereafter). In order to maintain repeatability across different testruns, we select a channel in the 5-GHz band free of external interference and set the MAC retransmission limit to 7 for both XPRESS and 802.11. We use Iperf to generate UDP traffic with 1470-byte payload packets and measure throughput as the goodput received at the flow destination.

We investigate the ability of XPRESS to exploit multi-path capabilities, in experiments where packets may travel different paths between the same source and destination, depending on the per-slot instantaneous differential queue backlogs. We set up a UDP flow between the farthest nodes in our testbed, and allow the XPRESS scheduler to use all possible links in the testbed. Figure 4(a) depicts the received throughput at the destination node versus the input source rate at the source node. The throughput of XPRESS increases linearly with the offered load until $5.5 \mathrm{Mbps}$, after which it remains stable at the maximum of 5.7 Mbps. On the other hand, 802.11 reaches only 3.5 Mbps (63\% gain for XPRESS) with a fixed rate of $24 \mathrm{Mbps}$ and $2.5 \mathrm{Mbps}$ (128\% gain for XPRESS) with auto-rate, after which throughput declines. The decline in 802.11 at high input rate occurs because of hidden terminal collisions along the 4-hop path, which trigger packet retransmissions and reduce the end-to-end throughput. XPRESS does not suffer from hidden terminals and is able to sustain the maximum throughput. We can also notice that 802.11 auto-rate offers less throughput than 802.11 with $24 \mathrm{Mbps}$ under high load, which is caused of collisions that often lead auto-rate to fall back to low PHY rates.

In addition, we investigate the delay properties of XPRESS. Figure 4(b) presents the cumulative distribution function (CDF) of the path hop count of each packet, while Figure 4(c) presents delay 
measurements obtained at the source. Figure 4(b) shows that, under high loads, almost all packets follow 3-hop or 4-hop paths, while as the load decreases an increasing fraction of packets follows longer paths. The reason is that queues are small and the differential backlogs are not effective in path differentiation; this is an inherent property of $B P$ scheduling. However, as shown in Figure 4(c), the delay of the slowest packets under $1 \mathrm{Mbps}$ load does not exceed $100 \mathrm{~ms}$, despite the long paths taken. Delays increase over the $5 \mathrm{Mbps}$ load, which is close to the capacity limit of $5.7 \mathrm{Mbps}$, as shown in Figure 4(a). Moreover, the delays are finite, which indicates that the congestion controller feeds the backpressure scheduler with rates within the network capacity region.

\section{Delay-Aware Network Utility Maximization on wnPUT Testbed}

The experimentation effort of the OPNEX team working at Poznan University of Technology (PUT) is based on experiments conducted on the local wnPUT testbed [4]. Due to the small scale of the wnPUT testbed, high controllability of the experimentation process is achieved that contributes to the research and evaluation cycles. The experimentation provided by PUT in OPNEX is based on scenarios concerning the Delay-Aware NUM System (DANUMS), and multi-path backpressure-oriented routing based on the OLSR protocol.

\section{A. The DANUM system}

The DANUM approach differs from existing NUM models for wireless multi-hop networks [2], which assume that the utility of each flow can be controlled, only in the case that the flow is able to adapt its rate at the transport layer (i.e., in the case of elastic TCP-like traffic). As a consequence, non-TCP flows (like streaming media CBR flows) are considered to be uncontrollable. In contrast to the abovementioned approaches, the DANUM system is aimed at applying delay-aware NUM-derived priorities (called 'urgencies') to both inelastic UDP-based streaming media flows and elastic TCP flows. The model is based on the fact that by controlling the priority of each flow the transmission delay is affected, which leads the flow's utility to change[10]. The delay-awareness of the DANUM framework results from the introduction of a new optimization variable, which enables the unification of utility definitions for both TCP and UDP flows [10].

The DANUM system implementation is based on two main architecture elements obtained from the DANUM problem decomposition. The first element is an indirect sender-side flow controller that is able to estimate flows' utilities (transmission quality) at real time, based on measurements of end-to-end throughput and end-to-end delay. The second element is a scheduling component, located above the 802.11 
MAC layer and aimed at providing the approximation of $B P$-based scheduling. The system operates above the MAC layer and does not change the standard wireless MAC 802.11 scheduling mechanism. More precisely, the DANUM system estimates and indirectly controls the layer-2 queue levels, trying to keep the MAC layer queue almost empty. As a result of capturing packets above MAC layer, the DANUM system is able to build and manage its own virtual queues and consequently provide the 'approximation' of Max-Weight Scheduling (MWS).

Additional signaling mechanisms are required to support the operation of the DANUM system, i.e., (i) the protocol for end-to-end delay and rate monitoring based on Delay Reporting Messages (DRMs), (ii) the protocol of queue level signaling based on Queue Reporting Messages (QRMs) and Urgency Reporting Messages (URMs), and (iii) the protocol enabling the estimation of MAC queue levels based on Layer-2 Queue Estimation Messages (L2QE). The DANUM system has been implemented as a loadable Linux Kernel Module that operates independently from the MAC layer scheduling and therefore is inter-operable with widely used protocols of the typical networking stack, such as TCP, UDP, IP, and 802.11 MAC. The implementation of the DANUMS shows that it is possible to implement an effective and easily deployable approximation of the MWS performed above the MAC layer.

Experiments on the DANUMS focus on scenarios, in which simultaneous service of file transferring and multimedia streaming is required. Selected results of experiments conducted in a 2-hop network (described in [10]) are presented in Figure 5. The statistics include end-to-end delay, rate, layer-2 queue levels (Q), and virtual queue levels (i.e., queue levels dependent from the delay-aware utility) - all corresponding to one TCP and three UDP flows served simultaneously in a DANUMS-controlled network. We notice in the subfigure that illustrates the evolution of resulting rate per flow that the bandwidth granted to the elastic TCP flow is reduced, as soon as the inelastic UDP3 flow starts, because they cannot be served simultaneously (due to the network capacity limit). DANUMS was also tested in IMS-based audiovisual streaming scenarios [11]. The experimental results confirmed the ability of ensuring 'fair coexistence' of media streams and file transfers and showed that DANUMS may be used to realize 'soft' admission control, and to increase the overall network utility.

\section{B. Multi-path and Back-Pressure OLSR extensions}

Proactive routing protocols, such as the Optimized Link State Routing (OLSR) Protocol, are able to pre-provision paths throughout the network, which in turn may be used as a basis for advanced network resource allocation, such as MWS. However, standard OLSR is a single-path protocol, while $B P$-based $M W S$ algorithms provide better network performance when used jointly with multi-path packet 
forwarding. Maintaining multiple paths towards each destination is a potential cause of routing loops, if packets switch paths en route in an uncontrolled fashion. In addition, $B P$ scheduling offers effective means for routing loop avoidance, when packets are transmitted along multiple paths: monitoring backlog levels on the path from the source to the destination may be used to avoid routing decisions that result in loops or backward packet forwarding.

Based on these observations, we proposed a multi-path extension of the OLSR protocol, specified in [12], which enables OLSR to effectively discover and maintain multiple paths towards each destination in the network. In addition, we proposed a MANET traffic engineering extension of the OLSR protocol, specified in [13], which leverages the multiple paths provided by [12]. This constellation of novel IETF specifications stem from implementations of $B P$ mechanisms and extensive experiments thereof using OLSR both on the small-scale wnPUT testbed, and on the larger DES-Testbed platform, and has proven to balance and increase end to end throughput in multiple MANET scenarios.

\section{LONG-Term ENERgy EFficient EnVironmental Monitoring on DES-Testbed}

\section{A. Long-Term Environmental Monitoring}

In this section, we demonstrate the main results of the project research applied to the field of environmental monitoring. During the Wireless Energy-Aware mulTi-Hop sEnsor Reading (WEAtHeR) experiment, the environment around the sensor nodes is monitored by gathering temperature, humidity and energy consumption measurements. These measurements are gathered periodically in an interval of 60 seconds and the samples are locally stored at the testbed's server.

The experimental setup consists of 5 specified source nodes that broadcast their samples and one wireless sink node that logs received sensor readings. A gossiping routing algorithm is used, so that all nodes, excluding the sink node, relay received messages with a probability $p \in[0,1]$ if they have not already received it. With $p$, the forwarding behavior of the routing nodes is controlled. Choosing a small value for $p$ results in less transmissions and thus in lower energy consumption. However, fewer samples are expected to be received by the sink, since packets are forwarded less often. During the runtime of the experiment, we studied different values for $p$, in order to evaluate the trade-off between data completeness and energy consumption.

A sample of 3-month results, presented in Figure 6, shows that a higher forwarding probability $p$ leads the sink node to receive a higher percentage of broadcasted messages. However, a higher forwarding probability increases the network-wide number of transmissions and consequently the energy consumption. Therefore, the light-weight implementation of the gossiping routing algorithm is appropriate for 
energy-aware networks to control the trade-off between energy usage and the overall rate of successfully received messages. Another interesting result is that even with a forwarding probability of $p=1.0$, the reception of a particular message by all network nodes can not be guaranteed. Finally, the experiment demonstrated the feasibility of measuring the energy consumption of WSNs accurately, which enables the energy efficiency evaluation of protocols proposed for low-power networks.

\section{B. DES-Testbed}

The Distributed Embedded Systems Testbed (DES-Testbed), which has been used for the purposes of the WEAtHeR experiment, is a hybrid wireless network located on the campus of Freie Universität Berlin [5] and currently comprises 120 indoor and outdoor DES-Nodes. Each DES-Node consists of a wireless mesh router equipped with one LogiLink WL0025 IEEE 802.11b/g USB NIC and two Compex WLM54SAG IEEE 802.11a/b/g Mini PCI cards based on the Atheros AR5414 chipset and one ScatterWeb MSB-A2 sensor node that uses frequencies between 863 and $870 \mathrm{MHz}$. Moreover, all sensor nodes are equipped with a Sensirion SHT-11 temperature and humidity sensor, as well as with a LTC4150 coulomb counter that provides accurate energy consumption measurements. For accessing the DES-Nodes, a testbed server (DES-Portal) functions as the central control instance and provides the databases used by the control framework (DES-Testbed Management System, DES-TBMS), which supports the definition, execution, and evaluation of experiments.

\section{CONClusions AND Future Work}

OPNEX delivered a first principles approach to bridge the gap between theory and experimentation by transforming the proposed algorithms into realistic ready-to-implement protocols that rely on advanced optimization theory principles. Through OPNEX, we proposed two different BP-inspired architectures, namely QLR and XPRESS, where the former is a simple load-aware routing algorithm that is also 802.11 compatible and significantly outperforms typical source-based routing schemes, while the latter is the first real TDMA-based implementation of the BP policy. Moreover, we proposed the DANUM framework, which is able to adapt the rate of non-TCP flows at the transport layer and thus significantly differs from existing NUM models for wireless multi-hop networks. Finally, we demonstrated the feasibility of measuring the energy consumption of WSNs accurately, through the execution of an environmental monitoring experiment. The resulting protocols were implemented and tested in the four wireless testbeds that were developed for the purposes of OPNEX project. The results obtained through intensive collaboration among the project partners, were rather encouraging in comparison with relevant state-of-the-art 
approaches and thus pave the way to further elaboration on implementation of more composite protocols in the future.

\section{ACKNOWLEDGMENTS}

This work was supported by the European Commission OPNEX STREP project (FP7-224218).

\section{REFERENCES}

[1] L. Tassiulas and A. Ephremides. "Stability properties of constrained queueing systems and scheduling policies for maximum throughput in multihop radio networks". IEEE Transactions on Automatic Control, 1992.

[2] L. Georgiadis, M.1 Neely, and L. Tassiulas. "Resource Allocation and Cross-Layer Control in Wireless Networks". 2006.

[3] NITOS Testbed, http://nitlab.inf.uth.gr/NITlab/index.php/testbed.

[4] Andrzej Szwabe, Pawel Misiorek, Adam Nowak, and Jacek Marchwicki. "Implementation of backpressure-based routing integrated with Max-Weight Scheduling in a wireless multi-hop network". In IEEE 35th Conference on Local Computer Networks (LCN), pages $983-988,2010$.

[5] Bastian Blywis, Mesut Guenes, Felix Juraschek, and Jochen Schiller. "Trends, Advances, and Challenges in Testbed-based Wireless Mesh Network Research". Mobile Networks and Applications, 15:315-329, 2010.

[6] J. Bicket, D.1 Aguayo, S. Biswas, and R. Morris. "Architecture and Evaluation of an Unplanned 802.11b Mesh Network". In Proc. of Mobicom, 2005.

[7] Iperf: The TCP/UDP Bandwidth Measurement Tool, http://dast.nlanr.net/Projects/Iperf/.

[8] R. Laufer, T. Salonidis, H. Lundgren, and P. LeGuyadec. "XPRESS: A Cross-layer Backpressure Architecture for Wireless Multi-hop Networks". In Proc. of ACM MobiCom, Las Vegas, NV, USA, Sep. 2011.

[9] D. Koutsonikolas, T. Salonidis, H. Lundgren, P. LeGuyadec, C. Hu, and I. Sheriff. "TDM MAC Protocol Design and Implementation for Wireless Mesh Networks". In Proc. of ACM CoNEXT, Madrid, Spain, Dec. 2008.

[10] Andrzej Szwabe, Pawel Misiorek, and Przemyslaw Walkowiak. "Delay-Aware NUM System for Wireless Multi-hop Networks". In Proceedings of IEEE European Wireless 2011 (EW2011), pages 530-537, Vienna, Austria, April 2011.

[11] A. Szwabe, P. Misiorek, and P. Walkowiak. "IMS-Based performance analysis of a MANET controlled by the DelayAware NUM system". In Proc. of IEEE Wireless Communications and Networks Symposium (WOCC2011 - Wireless), NJIT, Newark, New Jersey 07102, USA, April 2011.

[12] A. Szwabe, A. Nowak, E. Baccelli, J. Yi, and B. Perrein. "Multi-path for Optimized Link State Routing Protocol version 2". https://datatracker.ietf.org/doc/draft-szwabe-manet-multipath-olsrv2/, 2011. Work in progress.

[13] A. Szwabe, P. Misiorek, M. Urbanski, and E. Baccelli. "OLSRv2 Backpressure Traffic Engineering Extension". https://datatracker.ietf.org/doc/draft-szwabe-manet-backpressure-olsrv2/, 2011. Work in progress. 


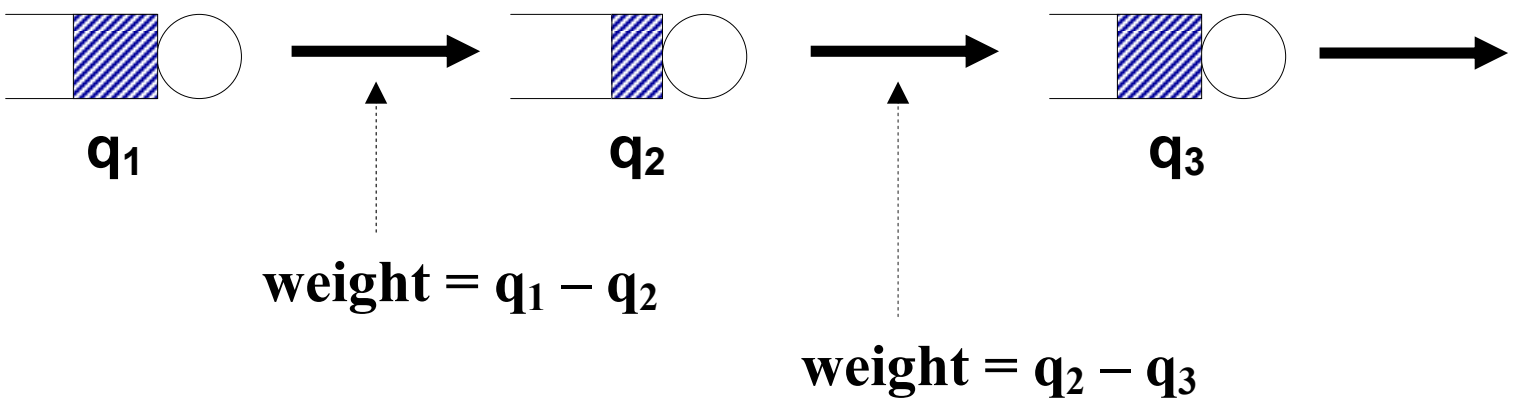

Figure 1: The underlying principle of the Back-Pressure policy. 


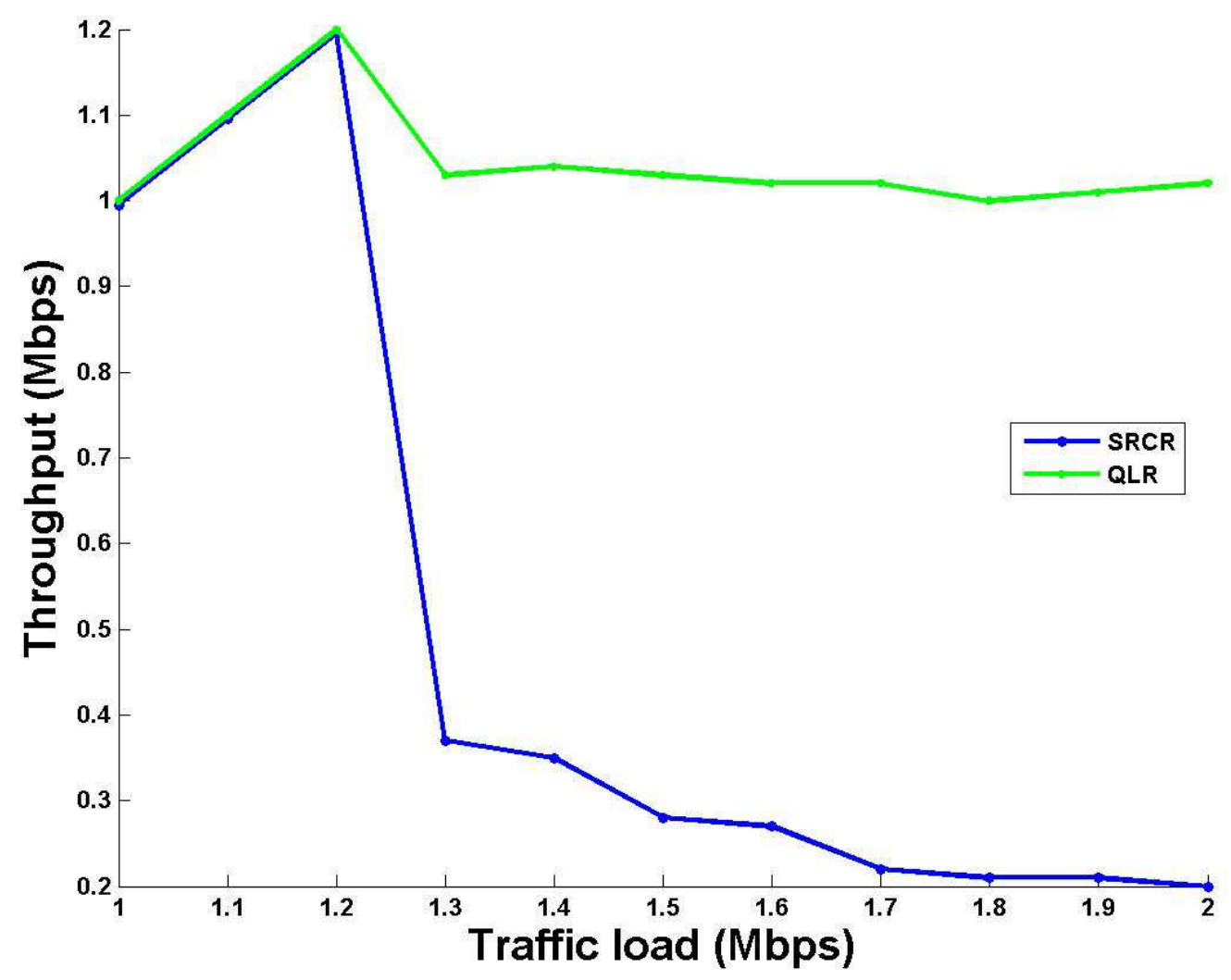

Figure 2: Throughput versus the input traffic load in the 5-node network. 


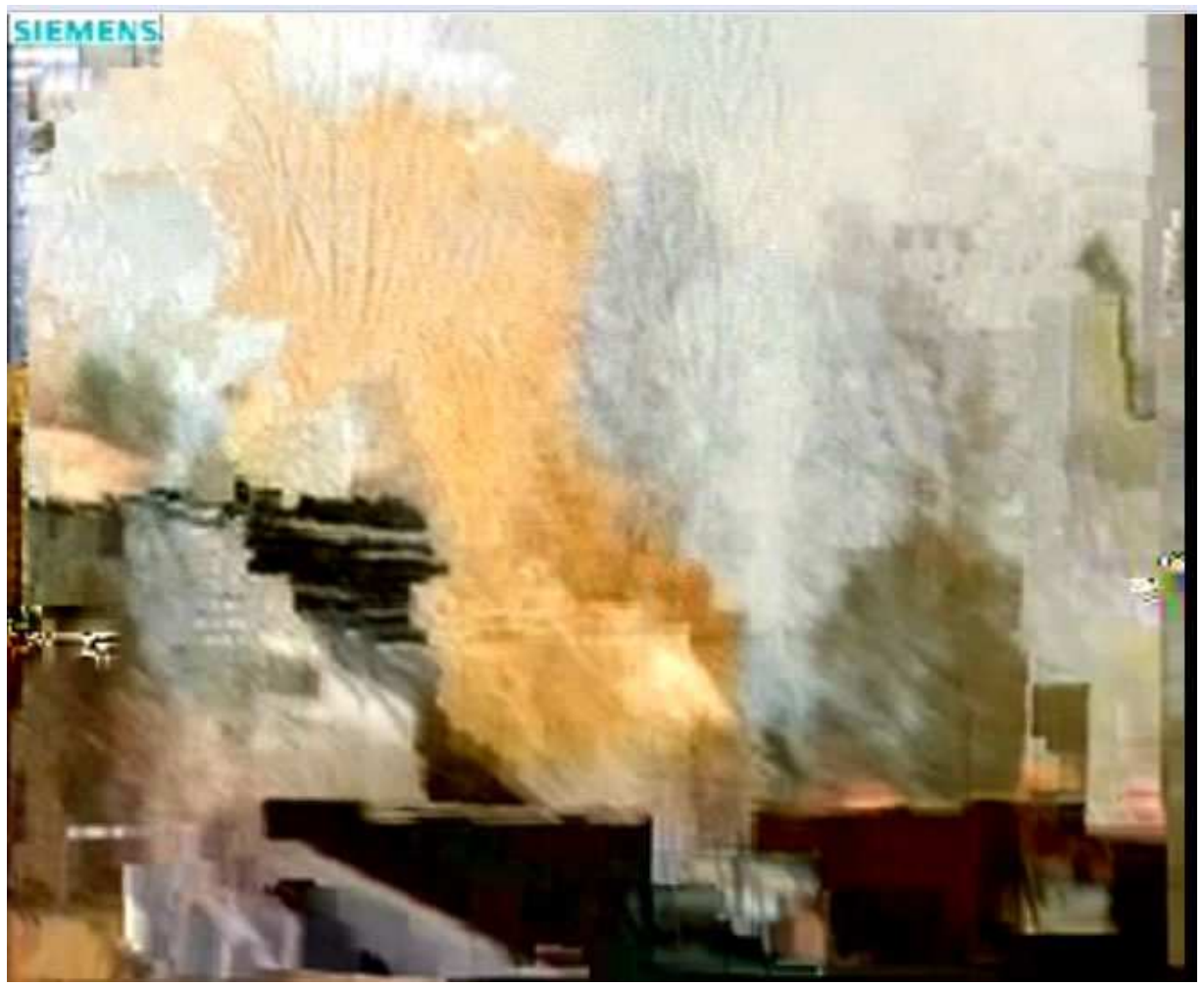

(a) SRCR screenshot

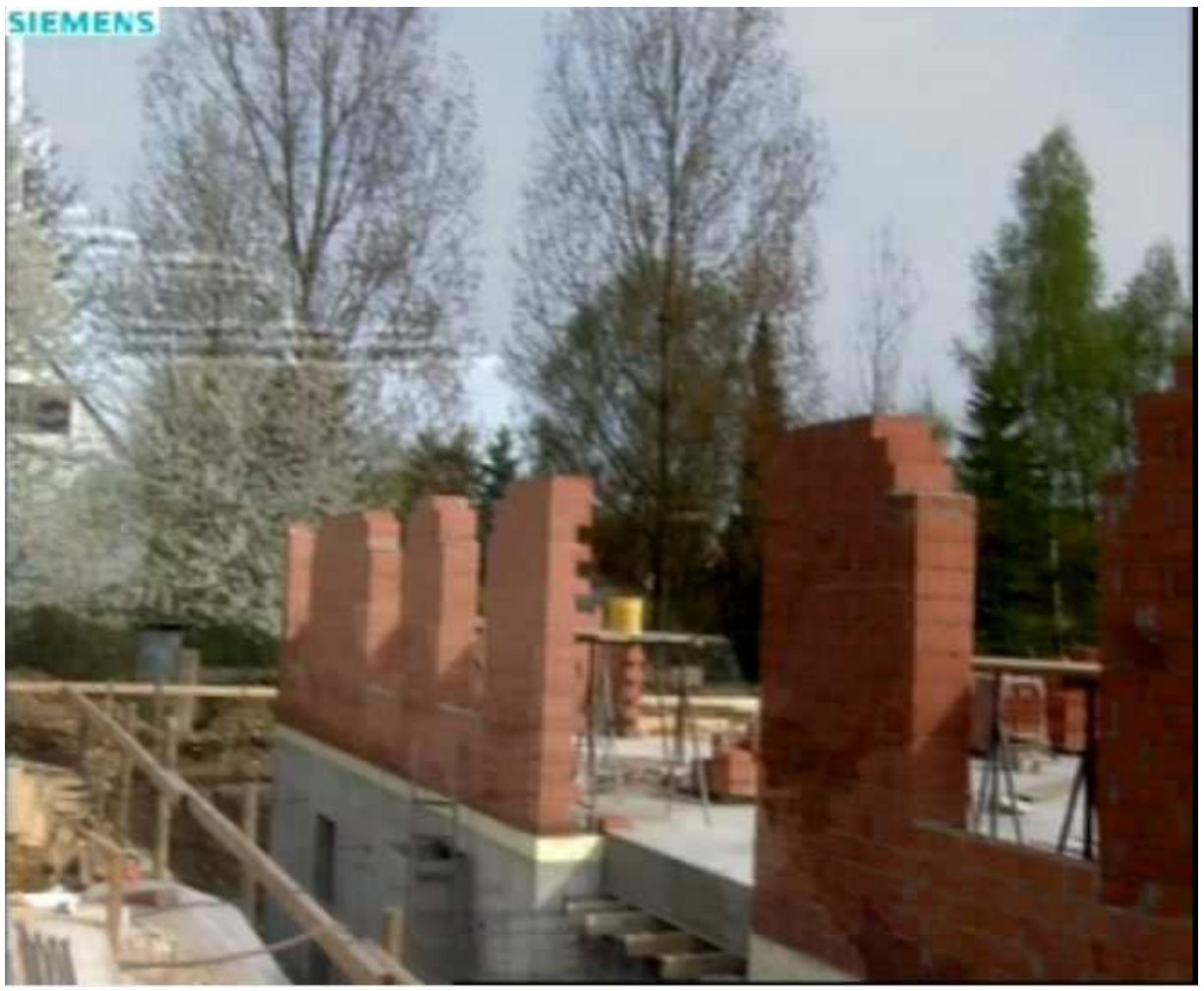

(b) $Q L R$ screenshot

Figure 3: Screenshots of two different frames, as transmitted according to the two approaches, SRCR and QLR 


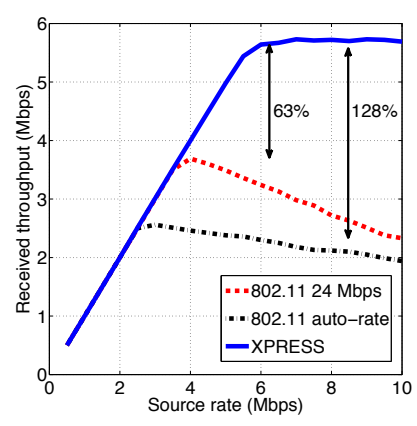

(a) Throughput at the receiver.

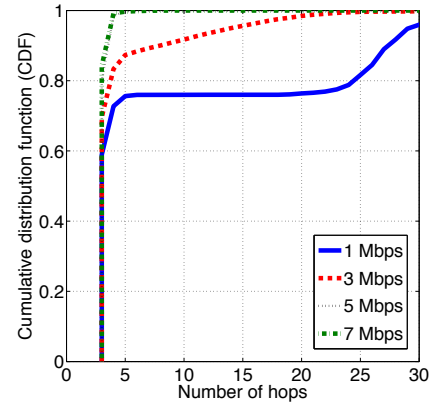

(b) Number of hops per packet.

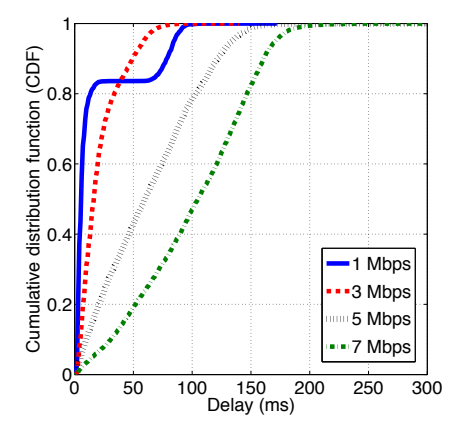

(c) Network delay.

Figure 4: The throughput, number of hops, and delay for the multi-path experiment. 

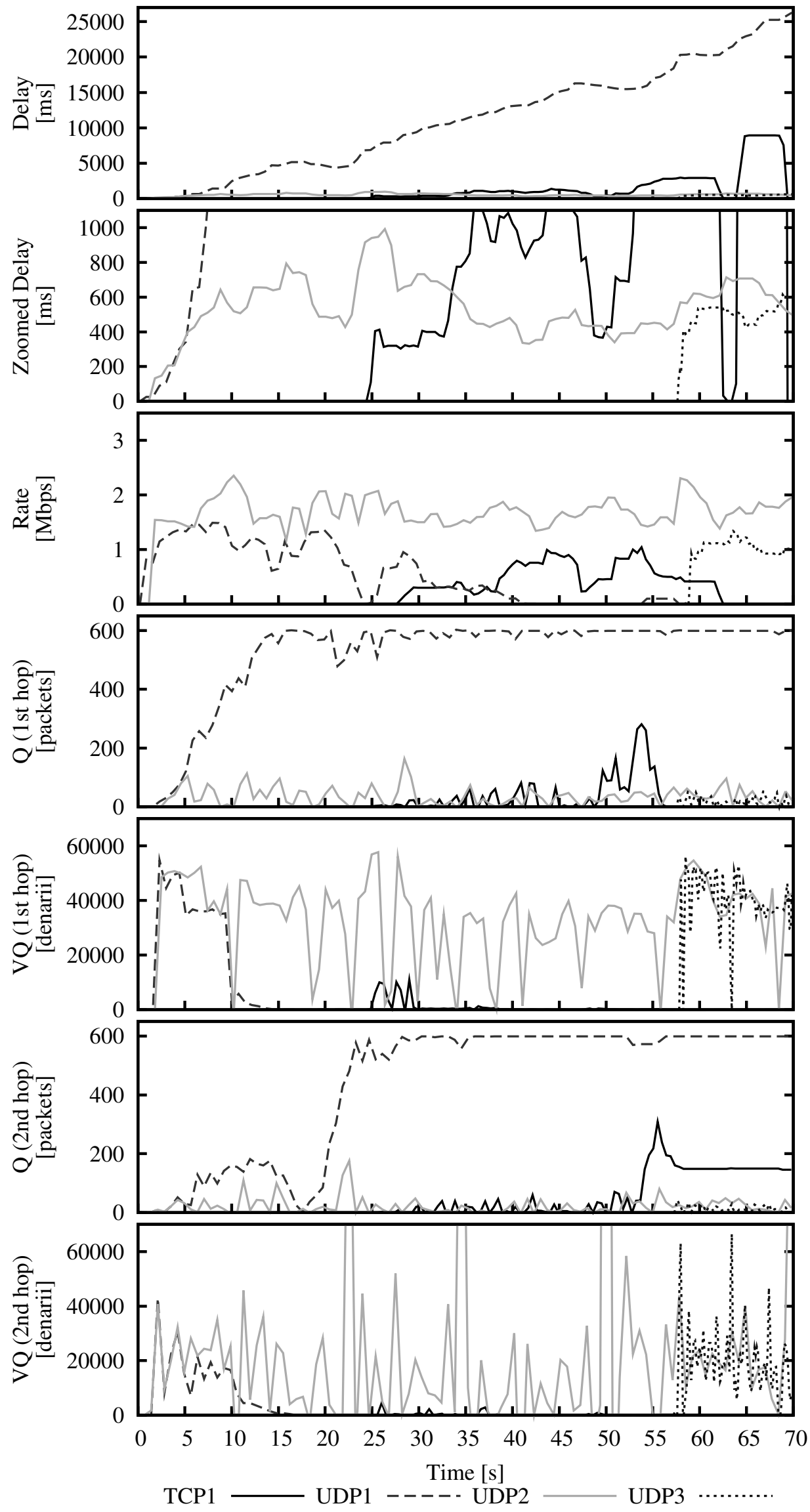

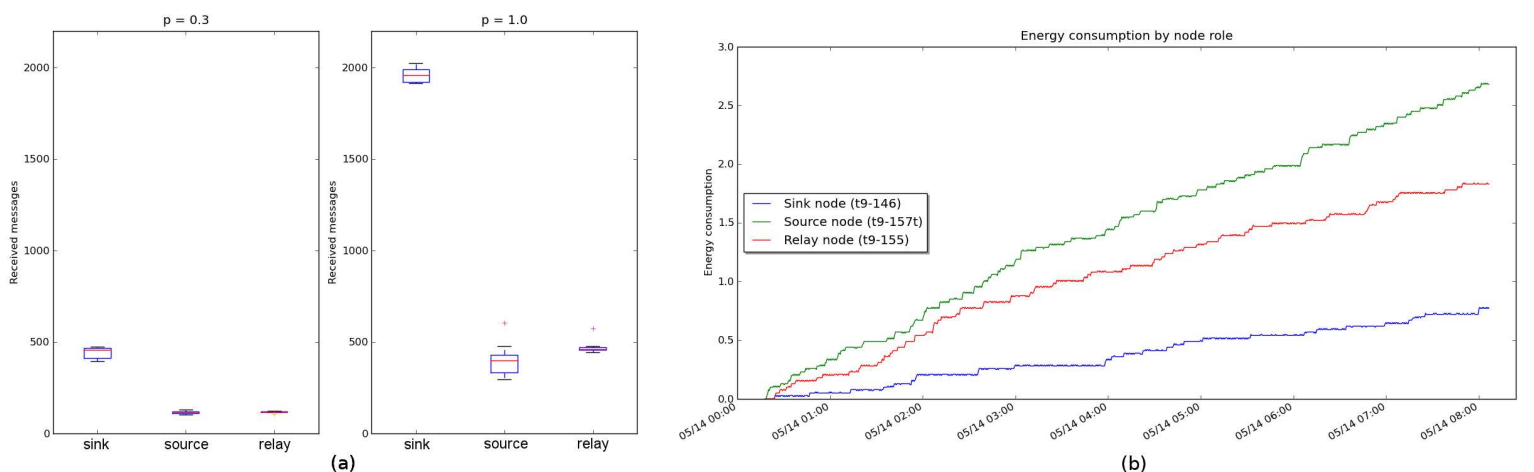

Figure 6: Results of the WEAtHeR experiment. In (a), the number of received messages per node role in 20 experiment replications is depicted. It shows as expected, that with a higher forwarding probability, more messages are received at the sink. In (b) the energy usage per node role based on the coulomb counter is displayed. 